Original Article

\title{
IMMUNOHISTOCHEMICAL IDENTIFICATION AND DISTRIBUTION OF GLUTAMATERGIC NMDA AND mGlu1 RECEPTORS IN THE PONTINE INTERTRIGEMINAL REGION IN RATS
}

\author{
Milan Stoiljkovic \\ Department of Pharmacology and Toxicology, University of Niš, Faculty of Medicine, Niš, Serbia
}

\begin{abstract}
Local glutamate simulation of intertrigeminal region (ITR) in the lateral pons evoked immediate cardiovascular and respiratory effects proposing its role in central cardiorespiratory control. Since pharmacological studies provided only functional evidence for the existence of glutamate receptors in the ITR and thereby specifying putative neurochemical substrate involved in this control, here we employed immunohistochemistry to examine expression and distribution of NMDA and mGlu1 receptors in this structure. Thirty adult male Sprague-Dawley rats were perfuse-fixed, their brains frozen and cut into sequential series of $20 \mu \mathrm{m}$ thick sections through the ITR. Immunohistochemistry was performed using polyclonal antibodies against NMDA-NR1, NMDA-NR2A and mGlul receptors. Labeled neurons in the ITR were analyzed using light microscope and computerized image analysis system for quantification of relative immunoreactivity as the mean of integrated optical density (IOD), and counting the immunopositive cells. Light microscopic analyses demonstrated NMDA-NRI-immunoreactivity mainly localized in the neuronal cell bodies with sparse distribution on primary dendrites, while NMDA-NR2A-immunoreactivity was basically somatically distributed. The mGlul-immunoreactivity was moderate and observed both in neuronal bodies and primary dendrites or extracellular matrix suggesting somatodendritic localization. Quantitative analyses of IOD showed very strong expression of NMDA-NR1, weak of NMDA-NR2A and strong-to-moderate expression of $m G l u R 1$, with differences in immunostaining signal distribution over rostro-caudal span of the ITR. Counting of immunopositive cells followed similar expression profile. Our data directly confirm the presence of glutamatergic NMDA and mGlul receptors in the ITR apparently involved in signaling pathways by which this region modulates cardiorespiratory functions such as blood pressure, heart rate and breathing.
\end{abstract}

Key words: intertrigeminal region, NMDA receptors, mGlul receptors, immunohistochemistry, rats.

\section{Introduction}

Dorsolateral pontine neurons located in the intertrigeminal region (ITR), parabrachial complex (PB) and Kölliker-Fuse nucleus (KF) are important components involved in cardiorespiratory coupling, a dynamic property of homeostasis involved in control of blood pressure, heart rate and breathing $[1,2]$. This coupling depends mainly on local circuitries and direct anatomical connectivity within these neurons and their inputs to forebrain structures involved in regulation of respiratory and cardiovascular functions [3, 4].

Previous studies posited particular role of glutamatergic neurotransmission for proper synchronization between breathing and cardiovascular dynamics as glutamate is shown to be essentially involved in both ascending and descending pathways of sympathetic respiratory and cardiovascular inputs [5]. In addition, evidence based on detection of mRNA by in situ hybridization suggests that a large proportion of the brainstem

Correspondence to: Milan Stoiljkovic, MD, Ph.D

Department of Pharmacology and Toxicology, University of Nis, Faculty of Medicine, University of Niš, Serbia, 81 Dr. Zoran Djindjić Blvd., 18000 Niš, Serbia

E-mail: mstoiljkovic@yahoo.com

Received February $5^{\text {th }}, 2019$, Accepted April $17^{\text {th }}, 2019$ neurons that contribute to respiratory and cardiovascular functions and presumably in their coupling are glutamatergic i.e. positive to vesicular glutamate transporter-2 [6, 7]. Indeed, it has been shown that local microinjections of glutamate into the PB, KF or ITR neurons elicit transitory cessation of breathing and increase in arterial blood pressure in anesthetized rats [1, 8-13]. While further neuroanatomical investigations of $\mathrm{PB} / \mathrm{KF}$ nuclei confirmed glutamate receptor subtypes involved in these effects [10,14-18], no morphological evidence exist to support presence of specific glutamate receptors in the ITR. In the set of previous pharmacological studies of ITR using subtype-selective antagonists of glutamate receptors only functional evidence for their existence therein was provided [12, 19-21]. Therefore, in the present study immunohistochemistry was performed using specific antibodies against NMDA (-NR1 and NR2A) and mGlu1 glutamate receptors in order to determine distribution and expression patterns of these receptors within the ITR. 


\section{Methods}

All experimental procedures were reviewed and approved by the local bioethics committee and conducted in compliance with the principles outlined in the EU and USA guidelines on the protection of animals used for scientific purposes (Directive 2010/63/EU and NIH Publications No. 80-23, revised 1996). Thirty adult male Sprague-Dawley rats (270-300 g) were kept individually in standard cages in a temperature and humidity-controlled environment under a 12:12 h light/dark cycle and with unlimited access to food and water. All efforts were made to minimize the number of animals used and their suffering.

To obtain brain tissue, animals were deeply anesthetized with the combination of ketamine $80 \mathrm{mg} / \mathrm{kg}$ and xylazine $5 \mathrm{mg} / \mathrm{kg}$ given intraperitoneally and transcardially perfused with $150 \mathrm{ml}$ of cold $0.9 \%$ saline containing $1 \mathrm{U} / \mathrm{ml}$ heparin, followed by $200 \mathrm{ml}$ of $4 \%$ freshly prepared paraformaldehyde in $0.1 \mathrm{M}$ phosphate buffer saline (PBS, pH 7.4) as a fixative. This method is consistent with the recommendations of the Panel on Euthanasia of the American Veterinary Medical Association. Immediately following perfusion, brains were removed from the skull, post-fixed in the same fixative for 2 hours, and then cryoprotected by immersion in $30 \%$ sucrose in $0.1 \mathrm{M}$ PBS at $4{ }^{\circ} \mathrm{C}$ for several days. For tissue processing the brainstem blocks were frozen and cut into an sequential series of $20 \mu \mathrm{m}$-thick coronal sections using a cryostat microtome (Leica CM 1850, Nussloch, Germany) and collected consecutively into three serial screen-bottom trays immersed in cold $0.01 \mathrm{M}$ PBS $(\mathrm{pH}$ 7.4). Thus, for each rat, approximately 25 sections were collected and equally spaced in three series (about $60 \mu \mathrm{m}$ apart) for the entire pontine ITR. This allowed systematic examination of distribution of glutamate receptors' immunoreactivity in the rostro-caudal extent of the structure (-9.16 $\mathrm{mm}$ to $-9.80 \mathrm{~mm}$ from bregma), according to rat brain atlas [22]. One section from every group of serial sections was used for immunostaining against NMDA-NR1, -NR2A, or mGlu1a receptors, while the others serve as a specificity control of labeling or processed for cresyl violet Nissl staining to examine the morphological features of the ITR. All sections from each alternate series were processed under identical conditions (i.e. time, temperature, and concentration of reagents).

Immunohistochemistry was carried out using polyclonal rabbit anti-NMDA-NR1, polyclonal rabbit antiNMDA-NR2A and polyclonal goat anti-mGlu1a antibodies as well as corresponding secondary biotinylated antibodies, all purchased from Santa Cruz Biotechnology (Santa Cruz, CA, USA). Briefly, the free-floating sections were treated with $3 \% \mathrm{H}_{2} \mathrm{O}_{2}$ for 10 min to suppress endogenous peroxide activity, followed by $2 \%$ normal serum for $30 \mathrm{~min}$ at $37^{\circ} \mathrm{C}$ to block nonspecific binding sites. Then the sections were incubated with polyclonal anti-NMDA-NR1 (sc-9058), anti-NMDANR2A (sc-9056) or anti-mGlu1a (sc-47130) antibodies at $4^{\circ} \mathrm{C}$ overnight. The primary antibodies were diluted
$1: 100$ in a carrier containing $2 \%$ normal serum in 50 $\mathrm{mM}$ Tris-buffered saline (TBS, $\mathrm{pH}$ 7.4). Thereafter, appropriate biotinylated secondary antibodies diluted $1: 300$ in the same carrier as primary antibodies, and avidin-biotin-peroxidase complex solution (Vectastain Elite Kit, Vector Laboratories, Burlingame, CA, USA) diluted 1:100 in TBS, were applied to the sections for $30 \mathrm{~min}$ each at room temperature. Between each of the steps, $50 \mathrm{mM}$ TBS (pH 7.4) with $0.05 \%$ Triton X-100 was used to thoroughly rinse the sections three times for 10 min by swaying. Immunoreactivity was detected by processing sections first with $0.05 \%$ 3,3-diaminobenzidine tetrahydrochloride (DAB, Sigma, St. Louis, MO, USA) in TBS-Triton ( $\mathrm{pH} \mathrm{7.7)} \mathrm{for} 5 \mathrm{~min}$, and then with addition of equal volume of DAB containing $0.01 \%$ $\mathrm{H}_{2} \mathrm{O}_{2}$ for the next $5 \mathrm{~min}$ at room temperature. The reaction was stopped by transferring the sections in ice-cold TBS and rinsing. Finally, the sections were mounted onto gelatin-coated slides, dehydrated, cleared, and sealed. Labeled sections were analyzed using Axio Observer Z1 microscope (Carl Zeiss, Göttingen Germany), linked to camera. Captured images were analyzed by computerized image analysis system (Image J, NIH, Bethesda, USA) for quantification of relative protein levels as the mean of integrated optical density (IOD) and for counting the immunopositive cells.

All antibodies used in the study (anti-NMDA-NR1, anti-NMDA-NR2A and anti-mGlu1a) were well characterized and their specificities established by the manufacturer. The rabbit polyclonal anti-NMDA-NR1 and antiNMDA-NR2A antibodies are purified immunoglobulins raised against amino acids $19-318$ or 23-76 mapping within an extracellular domain of human NMDA-NR1 or NMDA-NR2A, respectively. By Western blot analysis, anti-NMDA-NR1 antibody specifically yielded single bands between 100 and $150 \mathrm{kDa}$ in mouse brain extract. Specificity of anti-NMDA-NR2A antibody was confirmed by a single band at the expected molecular size $(200 \mathrm{kDa})$ in Western blots of $\mathrm{H} 4$ whole cell lysate. The goat polyclonal anti-mGlula antibody is an affinity purified immunoglobulin raised against a peptide mapping within an extracellular domain of mGluR1 of human origin. These antibodies have also been successfully used by several groups (for NMDA receptors see: [23, 24]; for mGlu1a receptors see: [25, 26].

Set sections adjacent to those processed for immunohistochemistry were used to verify the specificity of the labeling. This was achieved by running some slides in parallel through the entire procedure with the omission of the primary antibodies. No staining was observed in these control sections. As an additional control, the staining pattern obtained in this study was compared with previously published data on the distribution of NMDA-NR1 and mGluR1a immunoreactivity in the distinct brain regions. For instance, our NR1stained sections shown strong immunoreactivity in the hippocampus and hypothalamus as found by Petralia et al. [27], whereas strong mGluR1a-immunoreactivity was exhibited in the cerebellar cortex (molecular and Purkinje 
cell layers), as reported by Baude et al. [28]. The third series of the sections were processed with Nissl cresyl violet method in order to assess the cytoarchitectonic boundaries of the ITR using rat brain atlas [22].

For quantitative analysis of NMDA-NR1, NMDANR2A and mGlula receptor-like immunoreactivity in the ITR, the comparative sections were digitally photographed under the same exposure condition and analyzed using microscope-based image-analysis system ImageJ. Low power images ( $5 \mathrm{x}$ objective) were used to outline the ITR and order sections from rostral to caudal level relative to bregma. For each rat, an average of ten immunostained sections were sampled at a high magnification (40 x objective) and converted to binary images. To calculate the number of immunoreactive cells in each section and to measure the intensity of the immunoreaction by optical densitometry an unbiased counting frame of $100 \mu \mathrm{m} \times 100 \mu \mathrm{m}$ was used. This allowed assessment of changes in the number of expressing cells and the relative amount of the peptides. Approximately the same level of the ITR was chosen for every antibody and receptor-like immunoreactivity was consider positive for NMDA-NR1, -NR2A or mGlula if punctate staining was observed within the cell body or along the dendritic processes. To prevent multiple counting, neurons from every third evenly spaced section were included in the analysis and only immunopuncta inside the counting frame or touching its upper or right edge were counted. The counting was repeated at least twice for each section analyzed, which ensured that the number of profiles obtained was similar. Since light intensity can directly affect optical densitometric values for all of the measurements, lighting conditions were held constant all the time. Details regarding methodology for image processing and calibration were taken from publication by Jovanovic et al. [29].

Statistical analyses were done using one-way analysis of variance and Student's t-test. Data are expressed as mean \pm SEM, and difference consider significant if $p$ $<0.05$.

\section{Results}

Light microscopic analyses demonstrated a very strong homogenous positive staining in all sections investigated through different levels of ITR. Immunoreaction product was generally present in both cell bodies and proximal dendrites of labeled neurons as well as in the neuropil, mainly in dendritic processes. Immunoreactivity of NMDA-NR1 was predominantly located somatically since plasma membrane of many labeled neurons showed clearly visible immunoreaction product (Fig. 1). Fewer neurons also displayed NMDA-NR1
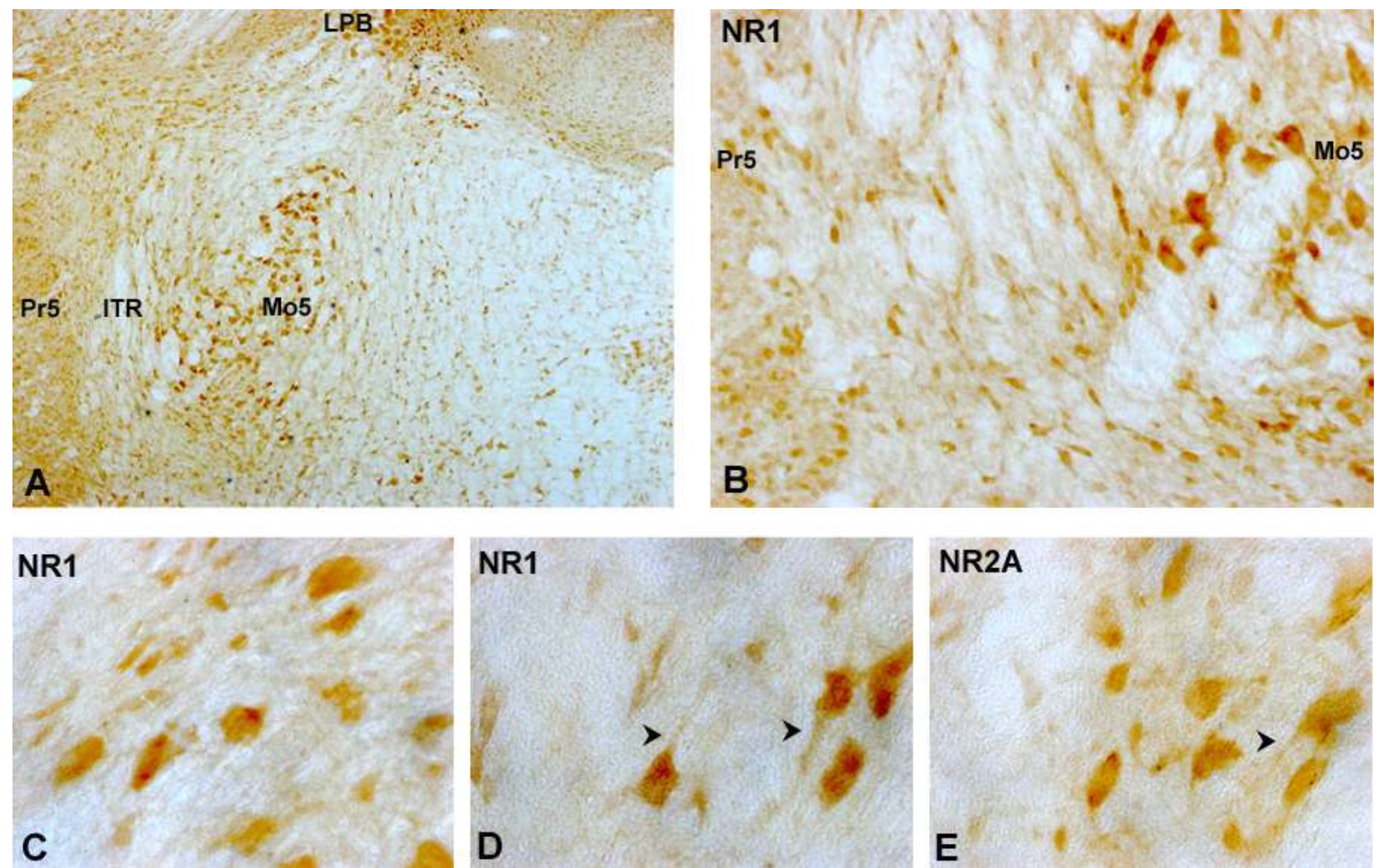

Fig. 1 Coronal sections of rat brain at the level of pontine ITR processed using specific antibodies against NMDANR1 and -NR2A receptors. Immunopositive reaction was detected in the cytoplasm of neurons and sparcely in the primary dendrites (arrowheads). Magnification (by objective): A (x 5), B (x 20), C-E (x 63). Abbreviations: ITR (intertrigenimal region), Pr5 (principal sensory trigeminal nucleus), Mo5 (motor trigeminal nucleus), LPB (lateral parabrachial complex nuclei). 

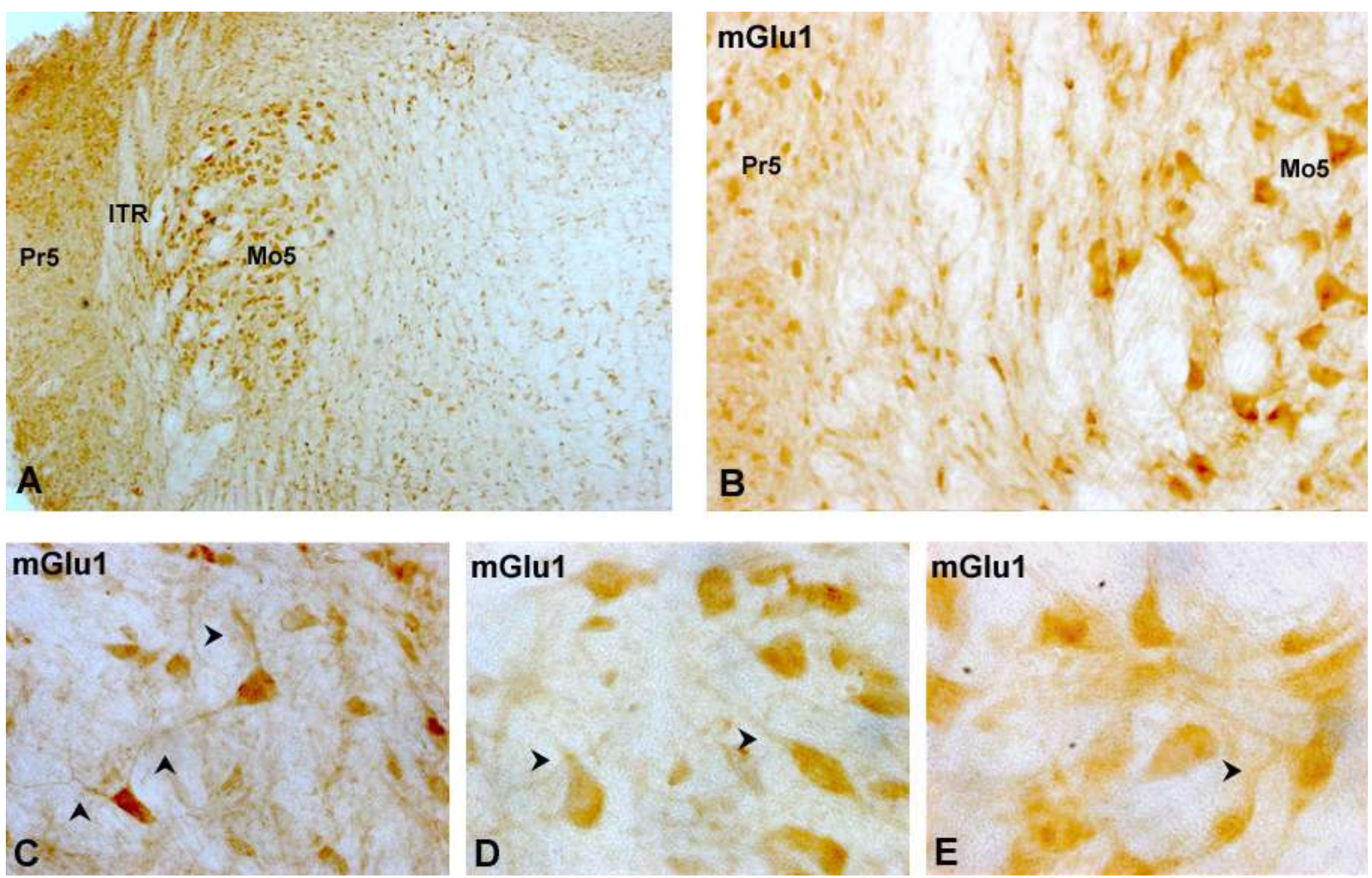

Fig. 2 Coronal sections of rat brain at the level of pontine ITR processed using specific antibodies against mGlu1 receptors. Immunopositive reaction was detected both in the cytoplasm of neurons and in primary dendrites (arrowheads). Magnification (by objective): A (x 5), B (x 20), C (x 40) D-E (x 63). Abbreviations: ITR (intertrigenimal region), Pr5 (principal sensory trigeminal nucleus), Mo5 (motor trigeminal nucleus).

positive immunoreaction on long thin profiles, which are presumably primary dendrites (Fig. 1C, D). At high magnification, NMDA-NR2A immunoreactivity consists of discrete products predominantly distributed in the soma of neurons scattered in the ITR (Fig. 1E). In mGlu1a labeled neurons immunoreactivity was rather homogeneously present both on primary dendrites and in the neuronal cell bodies (Fig. 2). In these sections, moderate fiber immunoreactivity with no apparent cellular staining was also noticed. Overall, both somatic and dendritic staining was observed (Fig. 2C, D, E). Control sections had no specific immunoreactivity above background (data not shown).

Quantitative analyses of immunostained sections at the same level of ITR from each rat revealed differences in NMDA-NR1, -NR2A and mGlu1a with respect to labeling intensity and numbers of labeled neurons. The most prominent staining intensity is for NMDA-NR1, moderate for mGlu1a and weak for -NR2A as measured by integrated optical density (Fig. 3). For both NMDANR1 and mGlu1, densest immunoreaction was distributed in medial part of the ITR, i.e. in sections extending from -9.30 to $-9.68 \mathrm{~mm}$ relative to bregma. In the most caudal sections, i.e. $-9.80 \mathrm{~mm}$ relative to bregma, apparently smaller numbers of neurons were stained for mGlu1 comparing to NMDA-NR1. Moreover, expression of the -NR2A immunoreactivity was significantly lower $(p<0.05)$ than for the other two receptor sub- types throughout the ITR. Similar results were obtained for counting immunoreactive cells for each receptor subunits analyzed at the same rostro-caudal level of the structure as summarized in Table 1.

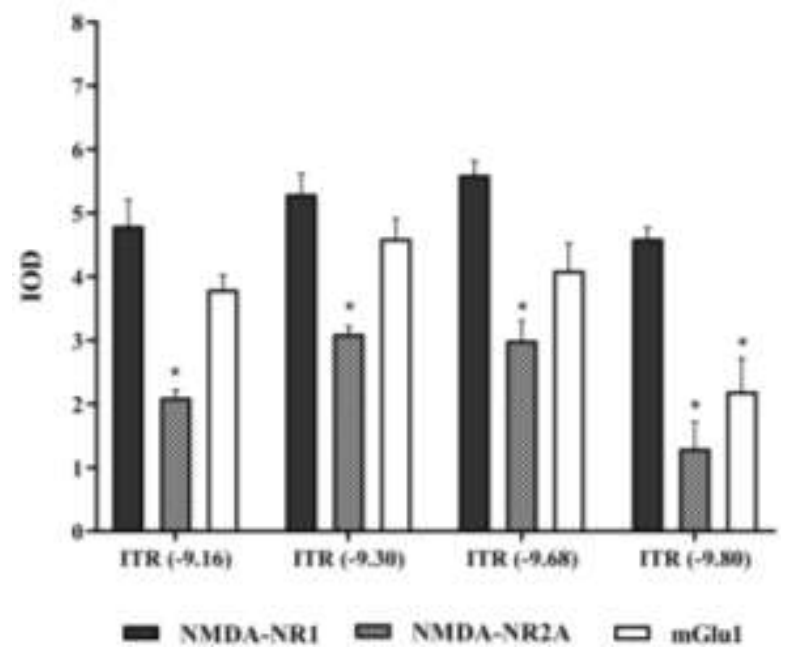

Fig. 3 Quantification of expression of NMDA-NR1, NMDA-NR2A and mGlu1 specific proteins using integrated optical density (IOD) at the level of ITR. Results are expressed as mean \pm SEM for IOD at every rostrocaudal level of every analyzed ITR sections $(* \mathrm{p}<0.05)$. 
Table 1 Number of immunopositive neurons for glutamatergic NMDA-NR1, NMDA-NR2A and mGlu1 receptors in the whole rostro-caudal dimension of the ITR.

\begin{tabular}{cccc}
\hline $\begin{array}{c}\text { Rostro-caudal } \\
\text { dimension of ITR }\end{array}$ & NMDA-NR1 & NMDA-NR2A & mGlu1 \\
\hline$-9.16 \mathrm{~mm}$ & $86 \pm 18$ & $21 \pm 17$ & $43 \pm 25$ \\
$-9.30 \mathrm{~mm}$ & $111 \pm 21$ & $35 \pm 18$ & $73 \pm 28$ \\
$-9.68 \mathrm{~mm}$ & $91 \pm 23$ & $26 \pm 12$ & $77 \pm 36$ \\
$-9.80 \mathrm{~mm}$ & $53 \pm 14$ & $28 \pm 14$ & $54 \pm 18$ \\
\hline
\end{tabular}

Results are expressed as mean \pm SEM immunopositive neurons in the rostro-caudal span of the ITR. Caunting sections were determined relative to bregma using rat brain stereotaxic atlas [22]. Overall lower number of NR2A immunopuncta was found in the ITR structure comparing to those for NR1 or mGlu1 receptors.

\section{Discussion}

Using light microscopic evaluation and IOD quantitative analyses of NMDA-NR1, -NR2A and mGlu1a immunopositive puncta, we showed here that glutamatergic NMDA and mGlu1 receptors are clearly expressed in the ITR. This is of particular importance since it represents the first direct confirmation of these receptors in the ITR, whose functional existence was previously suggested in a set of pharmacological studies with specific antagonists $[12,20,21]$. Moreover, our study revealed distribution of these receptors within the whole extent of ITR structure, as well as their specific localization at neuronal level that is necessary to delineate synaptic mechanisms involved in its cardiorespiratory control.

Previous immunohistochemical studies [23, 30] have shown that expression of -NR1 subunit could serve as a reliable marker of NMDA receptor presence in the brain since it is core component of the functional NMDA receptor complex. Our analysis of the ITR at the light microscopic level, showed that NMDA-NR1 and NR2A immunopositive puncta are predominantly located in neural cell bodies, with sparse neuropilar (at the primary dendrites) and extracellular matrix distribution within the ITR. On the other hand, mGlu1 immunopositivity was present on neural-dendritic sites since it is equally distributed both in neuronal cytoplasm and primary dendrites. Overall, the distribution of NMDA-NR1 subunit was quite similar to that of mGlula, however, the pericellular labeling that characterized some of the immunoreactive neuropil structures outlining the soma and proximal dendrites of ITR neurons was not encountered in the NR1-immunostained material. Furthermore, using quantitative IOD analysis of immunolabeled neurons with subtype-specific antibodies, we detected different degree of expression of these neurons within the ITR. More specifically, we found intense expression of -NR1, intense-to-moderate of mGlu1, and quite low expression for -NR2A proteins. These results further confirmed our light microscopic findings, and also suggest relatively higher density of NMDA comparing to mGlu1 receptors in the structure. Thus, ITR functional NMDA receptor complexes are most likely composed of NR1/NR2A subunits given their similar neuronal distribution. This aligns with the previous findings of similar NMDA complexes in the neighboring pontine structures, as well as with the evidence of their predominant expression in the pontomedullary region during postnatal development [31-33]. Based on histological and ultrastructural colocalization for -NR1 and -NR2A subunits in various neuronal populations, Petralia et al. [27] concluded that this type of NMDA receptor complex is mainly postsynaptically localized. Accordingly, NMDA receptors identified in our study are presumably postsynaptic receptors. Presence of mGlu1 immunopositivity in the ITR is also in correlation with previous study where their expression in lateral pontine $\mathrm{PB}$ and $\mathrm{KF}$ neurons was described [17]. However, exact synaptic localization of these receptors is difficult to reveal using conventional light microscopic examination, particularly when immunopositive puncta are visible on postsynaptic sites touching certain presynaptic elements, or when they are present on presynaptic sites which terminate on postsynaptic neuronal elements [34].

Importantly, these immunohistochemical findings corroborate our previous observations from pharmacological experiments, and additionally provide evidence for presence of NMDA and mGlu1 receptors in the ITR, which are apparently involved in its signaling pathways for neuromodulation of respiratory and cardiovascular functions. In these studies it has been shown that glutamate injected into the ITR can elicit immediate apnea, prolong vagal reflex apnea induced by serotonin injection [12, 20, 21], and increase systolic blood pressure [13]. Furthermore, local non-selective blockade of ITR glutamatergic receptors with kynurenic acid was able to suppress glutamate-induced central apnea and to increase reflex apnea evoked by systemic serotonin injection [12]. On the other hand, selective blockade of NMDA receptors in the ITR was enough to abolish glutamate central apnea [20], while local selective antagonism of mGlu1 prolonged reflex apnea without changing ITR response to glutamate stimulation [21]. Given these facts, presumed role of NMDA receptors, likely localized on somatic, postsynaptic sites in the ITR, is in mechanisms of dampening acute transitory perturbations in pontine cardiorespiratory centers, while mGlul receptors, distributed on somato-dendritic sites of its neurons, are involved in modulation of cardiorespiratory reflexes induced by excitations of vagal afferents.

In summary, this study provides direct evidence for the presence of glutamatergic NMDA and mGlu1 receptors in the ITR, and thereby add in defining synaptic mechanisms involved in its regulation of respiratory and cardiovascular homeostasis together with other pontine and ventrolateral medullar structures.

Acknowledgment: This work was supported by the Ministry of Science and Technological Development, Republic of Serbia grant 175092 . 


\section{References}

1. Dick TE, Baekey DM, Paton JF, Lindsey BG, Morris KF. Cardio-respiratory coupling depends on the pons. Respir Physiol Neurobiol 2009; 168:76-85.

2. Garcia AJ, 3rd, Koschnitzky JE, Dashevskiy T, Ramirez JM. Cardiorespiratory coupling in health and disease. Auton Neurosci 2013; 175:26-37.

3. Chamberlin NL, Saper CB. A brainstem network mediating apneic reflexes in the rat. J Neurosci 1998; 18:6048-6056.

4. Fulwiler CE, Saper CB. Subnuclear organization of the efferent connections of the parabrachial nucleus in the rat. Brain Res. 1984;319:229-259.

5. Zoccal DB, Furuya WI, Bassi M, Colombari DS, Colombari E. The nucleus of the solitary tract and the coordination of respiratory and sympathetic activities. Front Physiol 2014; $5: 238$.

6. Stornetta RL, Sevigny CP, Guyenet PG. Vesicular glutamate transporter DNPI/VGLUT2 mRNA is present in C1 and several other groups of brainstem catecholaminergic neurons. J Comp Neurol 2002; 444:191-206.

7. Stornetta RL, Sevigny CP, Guyenet PG. Inspiratory augmenting bulbospinal neurons express both glutamatergic and enkephalinergic phenotypes. J Comp Neurol. 2003;455:113-124.

8. Alheid GF, Milsom WK, McCrimmon DR. Pontine influences on breathing: an overview. Respir Physiol Neurobiol 2004; 143:105-114.

9. Chamberlin NL. Functional organization of the parabrachial complex and intertrigeminal region in the control of breathing. Respir Physiol Neurobiol 2004; 143:115-125.

10. Dutschmann M, Herbert H. NMDA and GABAA receptors in the rat Kolliker-Fuse area control cardiorespiratory responses evoked by trigeminal ethmoidal nerve stimulation. J Physiol 1998; 510 (Pt 3):793-804.

11. Lara JP, Parkes MJ, Silva-Carvhalo L, Izzo P, Dawid-Milner MS, Spyer KM. Cardiovascular and respiratory effects of stimulation of cell bodies of the parabrachial nuclei in the anaesthetized rat. J Physiol 1994; 477:321-329.

12. Radulovacki M, Pavlovic S, Saponjic J, Carley DW. Intertrigeminal region attenuates reflex apnea and stabilizes respiratory pattern in rats. Brain Res 2003; 975:66-72.

13. Topchiy I, Radulovacki M, Waxman J, Carley DW. Cardiorespiratory effects of intertrigeminal area stimulation in vagotomized rats. Brain Res 2009; 1250:120-129.

14. Dutschmann M, Guthmann A, Herbert H. NMDA receptor subunit NR1-immunoreactivity in the rat pons and brainstem and colocalization with Fos induced by nasal stimulation. Brain Res 1998; 809:221-230.

15. Guthmann A, Herbert H. Expression of N-methyl-D-aspartate receptor subunits in the rat parabrachial and Kolliker-Fuse nuclei and in selected pontomedullary brainstem nuclei. J Comp Neurol 1999; 415:501-517.

16. Guthmann A, Herbert H. In situ hybridization analysis of flip/flop splice variants of AMPA-type glutamate receptor subunits in the rat parabrachial and Kolliker-Fuse nuclei. Brain Res Mol Brain Res 1999; 74:145-157.

17. Guthmann A, Herbert H. Distribution of metabotropic glutamate receptors in the parabrachial and Kolliker-Fuse nuclei of the rat. Neuroscience 1999; 89:873-881.

18. Zidichouski JA, Easaw JC, Jhamandas JH. Glutamate receptor subtypes mediate excitatory synaptic responses of rat lateral parabrachial neurons. Am J Physiol 1996; 270:H1557-1567.
19. Isenovic ER, Radulovacki M, Carley DW. Impact of intertrigeminal region AMPA receptor blockade on respiratory responses in rats. Respir Physiol Neurobiol 2007; 158:39-44.

20. Radulovacki M, Stoiljkovic M, Saponjic J, Carley DW. Effects of intertrigeminal region NMDA and non-NMDA receptors on respiratory responses in rats. Respir Physiol Neurobiol 2007; 156:40-46.

21. Stoiljkovic M, Radulovacki M, Carley DW. Local antagonism of intertrigeminal region metabotropic glutamate receptors exacerbates apneic responses to intravenous serotonin. Respir Physiol Neurobiol 2009; 165:137-142.

22. Paxinos G, Watson C. The Rat Brain in Stereotaxic Coordinates. Fourth edition. Academic Press, San Diego. 1998.

23. Schlenker EH, Hansen SN. Comparison of NMDA modulation of breathing and NR1 expression in medullary nuclei of weanling male and female rats. Respir Physiol Neurobiol 2007; 155:203-212.

24. Wang LP, Li F, Wang D, Xie K, Wang D, Shen X, et al. NMDA receptors in dopaminergic neurons are crucial for habit learning. Neuron 2011; 72:1055-1966.

25. Hemstapat K, de Paulis T, Chen Y, Brady AE, Grover VK, Alagille D, et al. A novel class of positive allosteric modulators of metabotropic glutamate receptor subtype 1 interact with a site distinct from that of negative allosteric modulators. Mol Pharmacol 2006; 70:616-626.

26. Topolnik L, Azzi M, Morin F, Kougioumoutzakis A, Lacaille JC. mGluR1/5 subtype-specific calcium signalling and induction of long-term potentiation in rat hippocampal oriens/alveus interneurones. J Physiol 2006; 575:115-131.

27. Petralia RS, Wang YX, Wenthold RJ. The NMDA receptor subunits NR2A and NR2B show histological and ultrastructural localization patterns similar to those of NR1. J Neurosci 1994; 14:6102-6120.

28. Baude A, Nusser Z, Roberts JD, Mulvihill E, McIlhinney RA, Somogyi P. The metabotropic glutamate receptor (mGluR1 alpha) is concentrated at perisynaptic membrane of neuronal subpopulations as detected by immunogold reaction. Neuron 1993; 11:771-787.

29. Jovanovic I, Ugrenovic S, Antic S, Stefanovic N, Mihailovic D. Morphometric and some immunohistochemical characteristics of human choroids plexus stroma and psammoma bodies. Microsc Res Tech 2007; 70:617-627.

30. Hardingham GE, Bading $H$. The Yin and Yang of NMDA receptor signalling. Trends Neurosci 2003; 26:81-89.

31. Liu Q, Wong-Riley MT. Postnatal development of N-methylD-aspartate receptor subunits 2A, 2B, 2C, 2D, and 3B immunoreactivity in brain stem respiratory nuclei of the rat. Neuroscience 2010; 171:637-654.

32. Turman JE Jr., Ajdari J, Chandler SH. NMDA receptor NR1 and $\mathrm{NR} 2 \mathrm{~A} / \mathrm{B}$ subunit expression in trigeminal neurons during early postnatal development. J Comp Neurol 1999; 409:237-249.

33. Xing GG, Wang R, Yang B, Zhang D. Postnatal switching of NMDA receptor subunits from NR2B to NR2A in rat facial motor neurons. Eur J Neurosci 2006; 24:2987-2992.

34. Shigemoto R, Kinoshita A, Wada E, Nomura S, Ohishi H, Takada $\mathrm{M}$, et al. Differential presynaptic localization of metabotropic glutamate receptor subtypes in the rat hippocampus. J Neurosci 1997; 17:7503-7522. 